Z Herz- Thorax- Gefäßchir 2021 · 35:314-316 https://doi.org/10.1007/s00398-021-00455-w Angenommen: 13. August 2021

Online publiziert: 15 . September 2021

( $)$ Springer Medizin Verlag $\mathrm{GmbH}$, ein Teil von Springer Nature 2021

\section{Jane Somerville (geb. 1933)}

\author{
H. E. Ulmer \\ Heidelberg, Deutschland
}

Dieser Beitrag ist eine aktualisierte Fassung des Kapitels: Ulmer HE (2019) Meister ihres Fachs: Jane Somerville. In: Weil J, Kallfelz HC, Lindinger A, Schmaltz AA (Hrsg) Kinderkardiologie in Deutschland: 50 Jahre Deutsche Gesellschaft für Pädiatrische Kardiologie 1969-2019. Elsevier 2019, S. 290-291 (mit freundlicher Genehmigung des Elsevier Verlages).

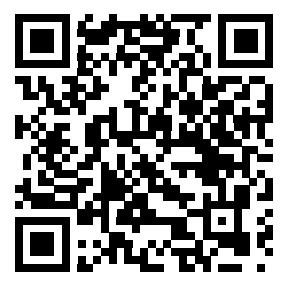

QR-Code scannen \& Beitrag online lesen
Erstmals zu Beginn des letzten Jahrzehnts zeigten medizinische Statistiken, dass die Zahl der Menschen mit einem angeborenen Herzfehler im Erwachsenenalter die der unter 18-Jährigen mit diesem Problem überstieg. Es ist zu erwarten, dass diese Entwicklung weiter zunehmen wird. Heute werden diese Patienten als sog. EMAH („Erwachsene mit angeborenem Herzfehler") oder international als sog. GUCH („grownups with congenital heart disease") bezeichnet. An der Beachtung und der angemessenen kardiologischen Versorgung dieser neuen Patientengruppe hat eine engagierte Kardiologin aus England den entscheidenden Anteil.

Jane Somerville (-Abb. 1) wurde am 24. Januar 1933 als Jane Platnauer im Londoner Stadtteil Kensington geboren. Den Vater, einen bekannten Theaterkritiker, erlebte Jane nur kurze Zeit, da er noch vor ihrem 6. Lebensjahr die Familie verließ. Der Mutter, eine ebenso bekannte Gesellschaftsreporterin mit engen Verbindungen zur Bloomsbury Group, verdankt sie ihr lebhaftes Temperament und ihre Courage. Drei Kriegsjahre verbrachte Jane aus Gründen der Sicherheit in einem Internat für Jungen in Nordwales: „The best education you could have if you want to enter in a male profession“. Schon 1947, nach dem Studium einiger alter Anatomiebücher ihrer Tante, einer Physiotherapeutin, stand ihr Entschluss fest, sich der Medizin zu widmen. Kurze Zeit danach, mit 16 Jahren, lernte sie als Nachbarn den Mann ihres Lebens kennen: den 20 Jahre älteren Kardiologen Walter Somerville, den sie dann 1957 als seine Studentin heiratete, mit dem sie vier Kinder haben und fast 50 Jahre glücklich zusammenleben sollte. Böse Zungen behaupten, dass

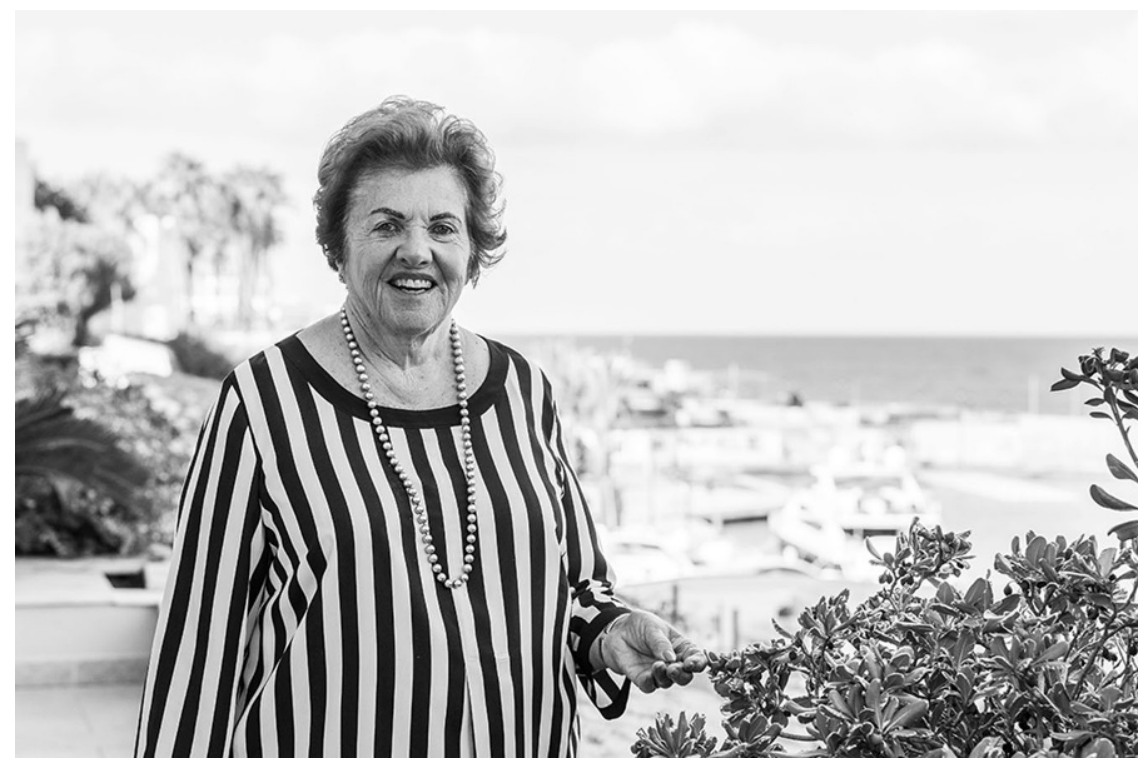

Abb. 1 ム Jane Somerville. (Aus TEMPLE Team [1]. Mit freundl. Genehmigung $\odot$ TEMPLE Magazine, Malta. Alle Rechte vorbehalten) 
Walter anfangs mehr an den Kochkünsten ihrer Mutter als an Jane selbst interessiert gewesen sein soll.

Noch während des Studiums an der Medical School des Guy's Hospital entdeckte sie nach einer Gastvorlesung von Alfred Blalock über die Chirurgie angeborener Herzfehler ihr Interesse für die Kardiologie. Nach ihrem Abschluss 1955 erhielt sie jedoch nur deshalb eine der begehrten Hilfsstellen im Guy's, weil sie die "Gold Medal in Clinical Surgery" gewonnen hatte. So wird sie chirurgische Assistentin von Sir Russell Brock, ist fasziniert von der Chirurgie angeborener Herzfehler, erkennt jedoch bald "... that my hands were not connected to my brain", und beendet ihre praktische chirurgische Karriere nach zwei Jahren.

1958 erhält Jane Somerville als erste Frau am renommierten Heart House Hospital eine Weiterbildungsstelle für Kardiologie. Es folgt eine aufregende Zeit zusammen mit Paul Wood, dem Genius der damaligen britischen Kardiologie, mit ihrem Mann Walter Somerville als verantwortlichem kardiologischem Consultant und etwas später mit dem speziell für angeborene Herzfehler zuständigen Herzchirurgen Donald Ross. Obwohl Paul Wood 1962 in seiner eigenen Klinik an einem akuten Herztod verstirbt, ausgelöst durch Kammerflimmern bei Infarkt, beginnt am Heart House in London eine neue kardiologische Ära mit internationalen Höchstleistungen. Auch im privaten Bereich entstehen lebenslange Verbindungen zwischen den Somervilles und Donald Ross. In ihrer spärlichen Freizeit knüpft Jane zusätzlich enge Verbindungen zum Hospital for Sick Children, in dem unter Richard Bonham-Carter und dem Chirurgen David Waterston eine spezielle Einheit zur Behandlung angeborener Herzfehler im Kindesalter etabliert worden war.

Die Entscheidung, vier Kinder zu haben, ermöglichte es Jane, jeweils eine kurze klinische Auszeit zu nehmen und etappenweise ihre Dissertation über atrioventrikuläre Septumdefekte zu schreiben, die sie nach zwei Schwangerschaften und fünf Jahren 1965 abschließen konnte.

Für eine reguläre Betreuung im Heart House waren Kinder mit angeborenen Herzfehlern zu jung und für die Great Ormond Street die Jugendlichen zu alt.
Zudem fehlte Jane ein pädiatrischer Abschluss. Allerdings bekam sie im Heart House, wo sie inzwischen als internistischer Consultant tätig war, die Genehmigung, ein entsprechendes Office einzurichten - allerdings auf ihre eigenen Kosten. Nach mehreren Gesprächen „... mit Lächeln und falschen Wimpern ..." bei einigen großzügigen anonymen Sponsoren konnte die kleine Einheit mit vier Betten 1975 in Betrieb genommen werden. Die Zahl der Zuweisungen von Patienten aus dem ganzen Land stieg rasch, und Janes zuverlässige Diagnosen erlaubten ihren chirurgischen Freunden Operationen mit hohen Erfolgsraten. Die wissenschaftliche Etablierung der Subspezialität „Kardiologie für angeborene Herzfehler" stieß jedoch sowohl bei den Pädiatern (AEPC) als auch bei den internistischen Kardiologen (ESC) damals auf größere Widerstände.

Es war noch 1975, als sie „... in ihrer Badewanne, ein Glas Champagner in der Hand ..." den Entschluss fasste, dass ein „Weltkongress für Pädiatrische Kardiologie und Chirurgie" für dieses Vorhaben hilfreich wäre, den sie jedoch notgedrungen wohl selbst auf die Beine stellen müsste. Große Hilfe hierzu erhielt sie vor allem von ihren Freunden, den großen Herzchirurgen aus nahezu allen Ländern der Welt. Nach vielen Mühen fand dieser 1. Weltkongress im Juni 1980 in London statt. Er hatte 1300 internationale Teilnehmer und stellt den Beginn der Erfolgsgeschichte einer gemeinsamen pädiatrischen, internistischen und chirurgischen Kardiologie für angeborene Herzfehler in jedem Lebensalter dar.

Ein empfindlicher Rückschlag für Jane war die Übernahme des Heart House durch das Royal Brompton Hospital 1989. Wegen der steigenden Zahl jugendlicher und junger erwachsener Patienten hatte sie noch kurzzuvor begonnen, einen speziellen Service für diese Gruppe aufzubauen, den sie auf zahlreichen internationalen Veranstaltungen, zu denen sie geladen war, modellhaft propagierte. Zu Hause im Brompton war sie jedoch ein zweites Mal gezwungen, einen derartigen Service auf eigene Kosten zu installieren. Mithilfe von Spenden ihrer Patienten und ihrer Freundin, der Opernsängerin Jessye Norman, konnte im Juli 1997 die „Jane Somerville GUCH
Unit" eröffnet werden: „I speak my mind. I'm often right - sometimes for the wrong reason - but I'm courageous."

Zwei Jahre später, 1999, wurde Jane, inzwischen Professorin für Kardiologie des Imperial College London, zwar in den Ruhestand verabschiedet, jedoch als Leiterin einer großen GUCH Unit an das Heart House berufen - den Ort, an dem einmal alles begonnen hatte.

Nun wurden Jane Somerville zahlreiche nationale und internationale Ehrungen zuteil. So wurde sie z.B. 2010 als zweite Frau nach Helen Taussig in die Hall of Fame der AEPC, der Europäischen Gesellschaft für Kinderkardiologie, aufgenommen. 2012 ehrte sie das American College of Cardiology (unter anderem neben Eugene Braunwald) als eine der "five legends of cardiovascular medicine".

Jane Somerville lebt heute abwechselnd in London und ihrer Wahlheimat Malta als Direktorin und Beraterin einer von ihr dort ins Leben gerufenen Klinik für GUCH-Patienten.

Korrespondenzadresse

Prof. Dr. H. E. Ulmer

Am Aukopf 5, 69118 Heidelberg, Deutschland herbert_ulmer@t-online.de

\section{Literatur}

1. TEMPLE Team (2020) If you want something done-ask a busy person! Professor Jane Somerville and her mission. https://www.templemagazines. com/post/if-you-want-something-done-aska-busy-person-professor-jane-somerville-andher-mission.Zugegriffen: 13. Aug. 2021 
Hier steht eine Anzeige.

黑 Springer 